\title{
Pruebas de función respiratoria, ¿cuál y a quién?
}

\author{
Respiratory function tests: which one and for whom?
}

\author{
Claudia Vargas-Domínguez,* Laura Gochicoa-Rangel,* Mónica Velázquez-Uncal,, Roberto Mejía-Alfaro,* \\ Juan Carlos Vázquez-García, ${ }^{*}$ Rogelio Pérez-Padilla, ${ }^{*}$ Luis Torre-Bouscoulet*
}

* Departamento de Fisiología Respiratoria, Instituto Nacional de Enfermedades Respiratorias Ismael Cosío Villegas, Ciudad de México.

\begin{abstract}
RESUMEN. Las pruebas de función respiratoria tienen aplicación clínica rutinaria en la práctica de la neumología. Son de utilidad en el diagnóstico de enfermedades respiratorias, permiten evaluar la respuesta a tratamientos, así como vigilar la progresión funcional y tienen, además, utilidad en la valoración del riesgo perioperatorio y en el pronóstico de diversas enfermedades. En este manuscrito revisamos, desde un punto de vista práctico, las pruebas de función respiratoria que consideramos son las más disponibles y las más útiles desde el punto de vista clínico. El propósito es auxiliar al clínico acerca de la prueba que debe solicitar de acuerdo con las características del paciente haciendo hincapié en que es conveniente evaluar, tanto la mecánica de la respiración como el intercambio gaseoso.
\end{abstract}

Palabras clave: Pruebas de función respiratoria, espirometría, pletismografía corporal, difusión de monóxido de carbono, caminata de seis minutos, prueba cardiopulmonar de ejercicio.

\author{
Abreviaturas: \\ A: Área \\ ATS: Sociedad Americana del Tórax \\ BODE: Índice multidimensional que incluye: índice de masa corporal, obstruc- \\ ción, disnea y capacidad de ejercicio \\ Cl: Capacidad inspiratoria \\ CO: Monóxido de carbono \\ CPT: Capacidad pulmonar total \\ CRF: Capacidad residual funcional \\ C6M: Prueba de caminata de seis minutos \\ CV: Capacidad vital \\ CVF: Capacidad vital forzada \\ CVL: Capacidad vital lenta \\ $\mathrm{DL}_{\mathrm{co}}$ : Difusión pulmonar de monóxido de carbono \\ $\mathrm{DL}_{c 0}$ sb: Difusión pulmonar de monóxido de carbono por técnica de respiración única \\ EPOC: Enfermedad pulmonar obstructiva crónica
}

\section{Correspondencia:}

\section{Dr. Luis Torre-Bouscoulet}

Departamento de Fisiología Respiratoria y Clínica de Trastornos

Respiratorios del Dormir. Instituto Nacional de Enfermedades

Respiratorias Ismael Cosío Villegas. Calzada de Tlalpan 4502, colonia

Sección XVI. México, D.F., 14080. Teléfono y fax: (52) (55) 56654748

Correo electrónico: luistorreb2001@yahoo.com.mm

Trabajo recibido: 18-III-2011; aceptado: 20-IV-2011.
ABSTRACT. Respiratory function tests have a routine clinical application in the practice of pneumology. They are useful in diagnosing respiratory diseases and make it possible to evaluate responses to treatments and to monitor functional progression. Also, they are important in evaluating perioperatory risks as well as prognoses in a variety of diseases. This article adopts a practical point of view in reviewing the respiratory function tests that we consider most easily available and most useful. The objective is to facilitate clinical physicians determine the test(s) they should request according to the characteristics of different patients. Finally, it emphasizes the fact that in most cases it is advisable to assess both respiration mechanics and the exchange of gases.

Keywords: Respiratory function tests, spirometry, body plethysmography, carbon monoxide diffusion, six-minute walking test, cardio-pulmonary exercise test.

FeON: Fracción espirada de óxido nítrico

G: Grosor de la membrana alveolocapilar

GA: Gasometría arterial

LIN: Límite inferior de la normalidad

L: Litro

P: Presión

$\mathrm{PaCO}_{2}$ : Presión parcial de bióxido de carbono en sangre arterial

$\mathrm{PaO}_{2}$ : Presión parcial de oxígeno en sangre arterial

$\mathbf{P}_{100}$ : Presión generada a los 100 milisegundos de la inspiración

$\triangle$ PCO: Diferencia de presión de monóxido de carbono

PCPE: Prueba cardiopulmonar de ejercicio

PDO: Prueba de desaturación de oxígeno

$\mathrm{Pe}_{\max }$ : Presión espiratoria máxima

PFR: Pruebas de función respiratoria

$\mathrm{Pi}_{\text {ax }}$ : Presión inspiratoria máxima

PTOS: Prueba de titulación de oxígeno suplementario

Q: Perfusión

$\mathbf{R}_{\text {int }}$ : Medición de resistencia por técnica de interrupción

$\mathrm{SaO}_{2}$ : Saturación de oxígeno en sangre arterial (medición gasométrica)

$\mathrm{SpO}_{2}$ : Saturación de oxígeno por oximetría de pulso

V: Volumen

$V_{\text {: }}$ Ventilación alveolar

VC: Volumen corriente

$V_{C O}$ : Producción de bióxido de carbono

VEF $_{1}$ : Volumen espiratorio forzado en el primer segundo

$\mathrm{VEF}_{1} \% \mathrm{p}: \mathrm{VEF}_{1}$ expresado como porcentaje del esperado o predicho

VER: Volumen espiratorio de reserva 
VGIT: Volumen de gas intratorácico

$\mathrm{VO}_{2}$ : Consumo de oxígeno

$\mathrm{VO}_{2}$ max: Consumo máximo de oxígeno

VPFE: Volumen pulmonar al final de la espiración

VR: Volumen residual

VVM: Ventilación voluntaria máxima

\section{INTRODUCCIÓN}

Existen diversas pruebas de función respiratoria (PFR) que son realizadas con diferentes propósitos. Desde un punto de vista práctico podemos clasificar a las PFR en pruebas de mecánica de la respiración, pruebas de intercambio gaseoso, pruebas de ejercicio (que exploran de manera integrada, tanto aspectos de la mecánica respiratoria como del intercambio de gases) y pruebas del control de la respiración. Dichas pruebas se realizan con fines clínicos, epidemiológicos, laborales o de investigación. ${ }^{1}$

Las PFR son indispensables en la práctica clínica neumológica. Son de utilidad en el diagnóstico de enfermedades respiratorias, permiten evaluar la respuesta a tratamientos, así como vigilar la progresión funcional y tienen, además, utilidad en la valoración del riesgo perioperatorio y en el pronóstico de diversas enfermedades. ${ }^{2}$ Por estos motivos, casi cualquier individuo evaluado por el especialista en medicina respiratoria es tributario para realizarle alguna de las PFR.

La decisión de cuál prueba debe ser realizada depende de la sospecha clínica; en general, es conveniente llevar a cabo una prueba que evalúe la mecánica de la respiración y otra el intercambio de gases. Lo anterior, debido a que ambas pruebas son complementarias y nos ayudan a explorar diferentes aspectos de la fisiología respiratoria. Las pruebas que con mayor frecuencia se utilizan para analizar la mecánica de la respiración son la espirometría, pletismografía corporal y pruebas de reto bronquial. Las de intercambio gaseoso son la difusión pulmonar de monóxido de carbono $\left(\mathrm{DL}_{\mathrm{CO}}\right)$, gasometría (usualmente arterial) y oximetría de pulso. Las pruebas de ejercicio permiten analizar la respuesta integrada del organismo (respuesta respiratoria, cardiovascular, metabólica, musculoesquelética y neurosensorial) a una situación de estrés; incluyen: prueba cardiopulmonar de ejercicio (PCPE) en protocolo incremental o de carga constante, prueba de caminata de 6 minutos (C6M), prueba de distancia corta y prueba del escalón, entre otras. ${ }^{3}$ Las pruebas del control respiratorio son rara vez necesarias y son principalmente utilizadas con fines de investigación.

En este documento revisamos las PFR de mayor aplicación clínica, haciendo hincapié en las indicaciones y utilidad de la prueba. ${ }^{1}$ No es el propósito agotar el tema en cada una de las pruebas ni enunciar los pasos necesarios para su ejecución, es una guía que puede ayudar al clínico a decidir la PFR más apropiada. Existen otras PFR que no se exponen en el presente escrito; sin embargo, el lector interesado puede revisar otros documentos que recomendamos ampliamente. ${ }^{1,4}$

\section{ESPIROMETRÍA}

La espirometría es la prueba más accesible y reproducible para evaluar la mecánica de la respiración. Mide la cantidad de aire que un sujeto es capaz de desplazar (inhalar o exhalar) de manera forzada en función del tiempo, lo que depende del calibre de los bronquios, de las propiedades elásticas del tórax y de los pulmones, así como de la integridad de los músculos respiratorios. Las principales mediciones de la espirometría son la capacidad vital forzada (CVF), el volumen espiratorio forzado en el primer segundo $\left(\mathrm{VEF}_{1}\right)$ y el cociente $\mathrm{VEF}_{1} / \mathrm{CVF}$. La CVF es el mayor volumen de aire, medido en litros (L), que se puede exhalar por la boca con máximo esfuerzo después de una inspiración máxima. El VEF ${ }_{1}$ es el volumen de aire exhalado durante el primer segundo de la maniobra de CVF. El cociente $\mathrm{VEF}_{1} / \mathrm{CVF}$ es la proporción de la CVF exhalada en el primer segundo de la maniobra de CVF.

La espirometría es el estándar de oro para medir la obstrucción bronquial y, por lo tanto, es de utilidad en el diagnóstico y seguimiento de diversas enfermedades respiratorias, como el asma o la enfermedad pulmonar obstructiva crónica (EPOC), entre otras. ${ }^{5}$ La espirometría permite también evaluar la respuesta a broncodilatadores o a estímulos que inducen obstrucción bronquial (ejercicio, metacolina, etc). ${ }^{6}$ La prueba es útil en el seguimiento de exposiciones laborales que pudieran afectar la función pulmonar, en la valoración del riesgo operatorio, para dictaminar incapacidad o impedimento y con fines de pronóstico. ${ }^{1}$

La restricción pulmonar no puede ser diagnosticada con precisión mediante una espirometría ya que ésta no permite medir el volumen residual (VR). A pesar de esta limitación, una vez diagnosticada apropiadamente la restricción pulmonar por los métodos adecuados (pletismografía corporal), la espirometría es de utilidad en el seguimiento de los pacientes con enfermedades restrictivas.

Existen algunas contraindicaciones relativas para la realización de la espirometría dentro de las que se incluyen: enfermedad cardiovascular aguda o descompensada en los últimos tres meses (infarto agudo del miocardio, insuficiencia cardiaca, enfermedad cerebrovascular), neumotórax en los noventa días previos, riesgo de hemoptisis o ruptura de aneurisma, cirugía de tórax, abdomen, ojos u oídos en los últimos tres meses, infecciones respiratorias agudas en las últimas dos semanas, tuberculosis pulmonar activa y embarazo avanzado o complicado. La espirometría no debe ser solicitada en pacientes con traqueotomía 
o sonda pleural ya que los resultados que se obtendrán no serán confiables.?

Antes de interpretar la prueba hay que verificar que exista la información suficiente para evaluar la calidad de la misma. Esta información incluye: nombre del paciente, datos generales (edad, género, peso y estatura), origen de los valores de referencia, los valores (CVF, VEF $\left.{ }_{1}, V E F_{1} / C V F\right)$ de tres maniobras aceptables y las tres gráficas de volumentiempo y flujo-volumen. Otros parámetros opcionales son la fecha de la última calibración, datos ambientales y algoritmo de interpretación.

Existen dos criterios básicos para analizar la calidad de una espirometría. ${ }^{7}$ Los criterios de aceptabilidad hacen referencia a que la maniobra tenga un inicio súbito, se inscriba el flujo-pico y exista un descenso gradual hasta la línea de base (Figura 1). La duración de la espiración forzada debe de ser al menos de 6 segundos, deberá alcanzar una meseta (variaciones de $<25 \mathrm{~mL}$ en el volumen espirado) al menos de un segundo y no debe de haber artefactos tales como esfuerzo variable, tos, cierre glótico, entre otros. La aceptabilidad de la maniobra se documenta en la curva flujo-volumen (permite evaluar principalmente el inicio de la maniobra) y la curva volumen-tiempo (evalúa especialmente el final de la maniobra) (Figura 1). Una vez obtenidos los tres esfuerzos aceptables (hasta un máximo de 15 esfuerzos), se procede a evaluar la repetibilidad. Mediante el análisis de la repetibilidad podemos saber qué tanto se parece un esfuerzo al otro. Recordemos que cuando un fenómeno es consistente existe menos posibilidad de error. La repetibilidad (la diferencia entre los valores de las dos mayores CVF y entre los dos valores mayores de $\mathrm{VEF}_{1 \text {, }}$ aunque provengan de maniobras diferentes) no debe de ser en condiciones ideales, mayor a $150 \mathrm{~mL}$ o a 100 $\mathrm{mL}$ cuando la CVF sea menor de $1 \mathrm{~L}$. Se acepta que la repetibilidad pueda ser hasta un máximo de $200 \mathrm{~mL}^{7}$
Los tres patrones funcionales que pueden identificarse en una espirometría son normal, obstructivo y sugerente de restricción. ${ }^{2}$ Como se ha expresado antes no existe el patrón mixto en la espirometría. Únicamente cuando se dispone de medición de volúmenes pulmonares puede identificarse dicho patrón funcional, el cual se define por obstrucción documentada en la espirometría y restricción documentada en la pletismografía.

Las estrategias de interpretación pueden ser revisadas extensamente en otros documentos. ${ }^{2}$ Brevemente mencionaremos que una vez que analizamos la calidad de la prueba, debemos enfocarnos en el cociente $\mathrm{VEF}_{1} / \mathrm{CVF}$ dado que es este parámetro y no el $\mathrm{VEF}_{1}$, el que permite identificar si existe un proceso obstructivo. El criterio más popular de un patrón obstructivo; pero no necesariamente el mejor, es cuando el cociente $\mathrm{VEF}_{1} / \mathrm{CVF}$ es menor a $70 \%$. Ese punto de corte es el internacionalmente aceptado; ${ }^{5} \mathrm{sin}$ embargo, nosotros recomendamos que se utilice el límite inferior de la normalidad ya que dicho cociente disminuye de manera normal conforme se incrementa la edad y si eso no se toma en cuenta podrían generarse falsos positivos. ${ }^{8}$ En la Tabla 1 se resumen los patrones funcionales.

Cuando existe un patrón obstructivo acompañado de disminución de la CVF, puede tratarse de un patrón mixto (obstrucción y restricción), o bien deberse a atrapamiento aéreo (pseudorrestricción). La única manera de distinguir entre ambas situaciones es mediante la medición de volúmenes pulmonares por lo que, insistimos, la espirometría no permite identificar un patrón mixto. La gravedad de la obstrucción debe valorarse mediante el $\mathrm{VEF}_{1}$ expresado como porcentaje del esperado (Tabla 2).

La respuesta al broncodilatador debe evaluarse de 20 a 30 minutos después de su administración (salbutamol 400 $\mu \mathrm{g})$. Se le llama respuesta positiva cuando observamos un cambio al menos de $200 \mathrm{~mL}$ y $12 \%$ en cualquiera de los parámetros espirométricos $\left(\mathrm{VEF}_{1}\right.$ y/o CVF). ${ }^{7}$ No olvidemos

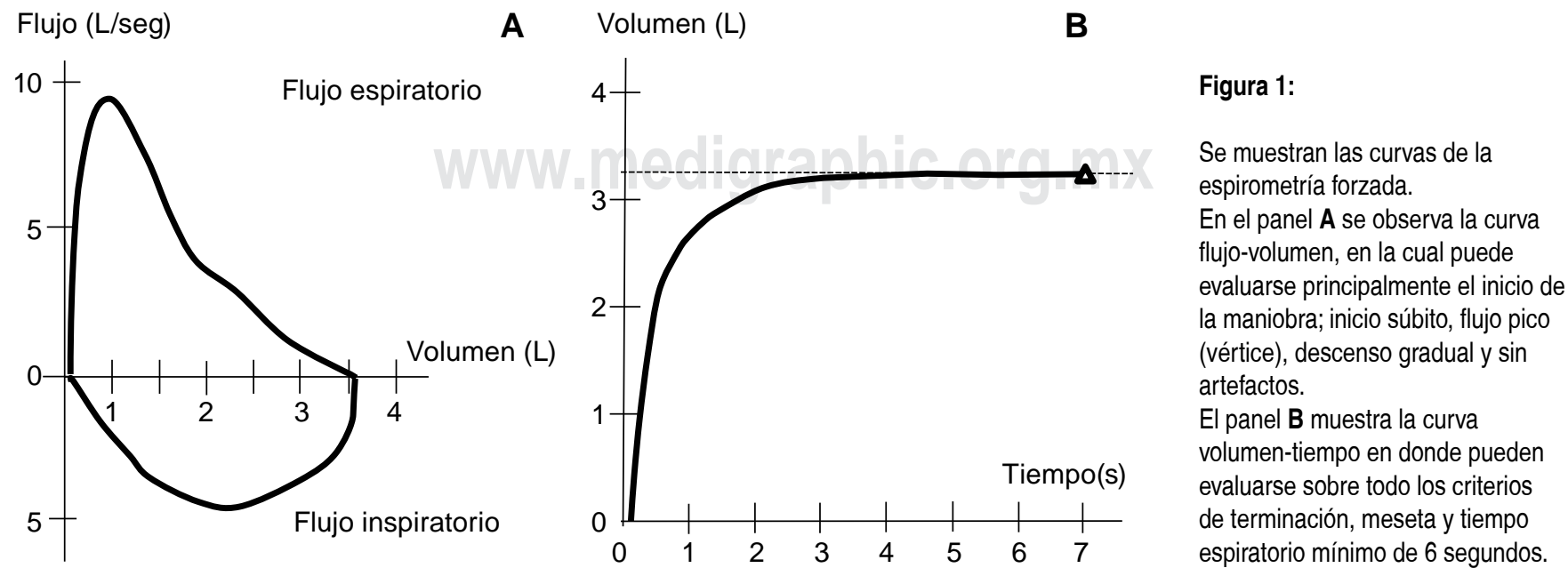


Tabla 1: Patrones funcionales en espirometria.

\begin{tabular}{|l|c|c|c|c|}
\hline Patrón funcional & VEF1/CVF & CVF & VEF1 & Observaciones \\
\hline Normal & $>70 \% 0>$ LIN & $>80 \%$ & $>80 \%$ & Ninguna \\
\hline Obstructivo & $<70 \% 0<$ LIN & $>80 \%$ & Cualquiera & Graduar la gravedad de la obstrucción \\
\hline Sugerente de restricción & $>70 \% 0>$ LIN & $<80 \%$ & Cualquiera & $\begin{array}{l}\text { Confirmar mediante la medición de } \\
\text { volúmenes pulmonares }\end{array}$ \\
\hline
\end{tabular}

$V_{\text {EF }}$ : Volumen espiratorio forzado en el primer segundo; CVF: capacidad vital forzada; LIN: Límite inferior de la normalidad.

Tabla 2: Gravedad de la obstrucción utilizando el volumen espiratorio forzado en el primer segundo expresado como porcentaje del esperado (VEF1 \% p) de acuerdo con la clasificación de la ATS.

\begin{tabular}{|l|c|}
\hline Grado de obstrucción & VEF1 \% $\mathbf{p}$ \\
\hline Leve & $80-100$ \\
\hline Moderado & $70-79$ \\
\hline Moderadamente grave & $50-69$ \\
\hline Grave & $35-49$ \\
\hline Muy grave & $<35$ \\
\hline
\end{tabular}

que cuando se analiza la respuesta al broncodilatador hay que interpretar tres aspectos de la prueba: 1) la espirometría inicial (prebroncodilatador), 2) la respuesta al broncodilatador y 3) la espirometría final (postbroncodilatador). Es frecuente observar pacientes que tienen una respuesta completa al broncodilatador, y que la espirometría posterior al broncodilatador sigue mostrando obstrucción; esto se observa en pacientes con asma y remodelación de la vía aérea o en pacientes con EPOC e hiperreactividad bronquial, entre otras patologías.

La espirometría también puede realizarse de manera relajada o tranquila, dicha variante recibe el nombre de espirometría lenta. La maniobra lenta es cómoda de realizar, no requiere de un esfuerzo físico extenuante y provee información adicional a la maniobra forzada. La primera fase de la espirometría lenta consiste en respiraciones a volumen corriente (VC) con el fin de alcanzar un nivel estable de volumen pulmonar al final de la espiración (VPFE); en la segunda fase, se hace una maniobra de inspiración máxima hasta llegar a la capacidad pulmonar total (CPT) para concluir con la tercera fase, que es una espiración relajada y completa con meseta de por lo menos un segundo (Figura 2). Otra forma de realizar esta prueba (que para algunos pacientes es más incómoda que la primera) es haciendo una maniobra de espiración máxima a partir del VPFE seguida de una inspiración máxima, y nuevamente una espiración completa y relajada. Las principales mediciones obtenidas a partir de la espirometría lenta son la capacidad vital lenta (CVL) y la capacidad inspiratoria (Cl). La CVL es

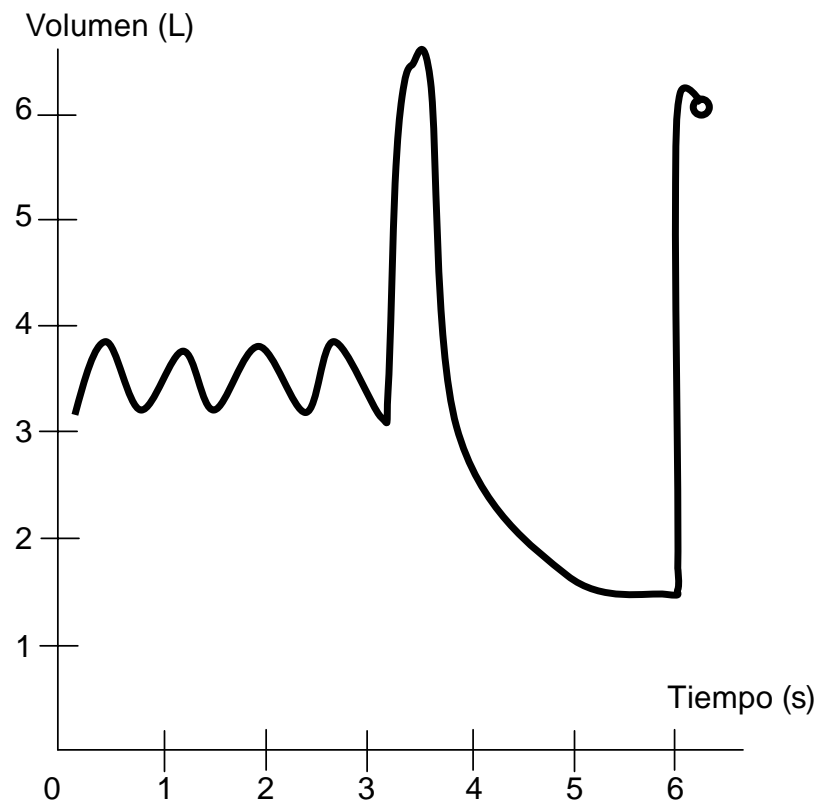

Figura 2: Espirometría lenta. En la primera parte de la gráfica se pueden observar respiraciones a volumen corriente, luego una maniobra de capacidad inspiratoria (inspiración máxima después de una espiración a volumen corriente), para finalmente realizar una espiración relajada y completa con meseta de por lo menos un segundo.

el volumen máximo de aire medido en $\mathrm{L}$, que puede ser espirado de una forma relajada después de una inspiración máxima. La $\mathrm{Cl}$ es el máximo volumen de aire que puede ser inspirado después de una espiración a VC. De manera complementaria a las mediciones obtenidas durante la maniobra forzada, la CVL y la $\mathrm{Cl}$ son de utilidad para evaluar la respuesta al broncodilatador con los mismos criterios de respuesta que en la espirometría forzada $(200 \mathrm{~mL}$ y 12\%). Aunque el estándar de referencia para la medición de la $\mathrm{Cl}$ es la pletismografía corporal, la obtenida mediante espirometría lenta resulta ser fácil de realizar y es de utilidad en la evaluación de la hiperinflación pulmonar y de la respuesta a diversos tratamientos. La principal limitación de la espirometría lenta es que es menos repetible que la maniobra forzada, está menos estandarizada y los valores de referencia disponibles son insuficientes. 


\section{PLETISMOGRAFÍA CORPORAL}

Esta prueba es considerada como el estándar de referencia para la medición absoluta de los volúmenes pulmonares ya que mide el volumen total de gas intratorácico; es decir, mide el volumen de aire en el tórax esté o no en contacto con la vía aérea; por ejemplo, el aire atrapado en bulas enfisematosas. ${ }^{9}$

La medición de volúmenes y capacidades pulmonares es indispensable en condiciones específicas que requieren de un diagnóstico fisiológico correcto. Estas condiciones incluyen: 1) medición de gas atrapado o atrapamiento aéreo, 2) establecer el diagnóstico de alteración restrictiva, 3) establecer el diagnóstico de alteración mixta (obstructiva y restrictiva), 4) en la valoración de riesgo quirúrgico, 5) evaluación de incapacidad laboral y 6) cuantificación del espacio aéreo no ventilado (se requiere de medición por dilución de helio).

Existen varios métodos para medir los volúmenes pulmonares. ${ }^{9}$ El que se utiliza con mayor frecuencia es la pletismografía corporal de volumen (V) constante. Esta prueba, como su nombre lo dice, implica que el volumen no cambia y las mediciones se llevan a cabo por cambios de presión (P) tomando en cuenta la Ley de Boyle en donde $P_{1} V_{1}=P_{2} V_{2}$. Esto se logra al introducir al sujeto a una cabina hermética que cuenta con dos transductores de presión. La incógnita en esa ecuación es $V_{1}$ que en realidad es la capacidad residual funcional (CRF) o volumen de gas intratorácico (VGIT). La CRF es la suma del volumen de reserva espiratorio (VER) y del VR y, en el contexto de la mecánica de la respiración, es el punto de equilibrio entre la retracción elástica del parénquima pulmonar y las fuerzas de oposición de la caja torácica. Es el volumen de aire que queda en los pulmones después de una respiración a VC.

Después de varias respiraciones a VC con el individuo en el interior de la cabina hermética, ocurre una oclusión que impide el flujo aéreo verdadero. Durante la oclusión, el paciente debe hacer una respiración conocida como jadeo con una frecuencia de 3-5 respiraciones por minuto. La oclusión dura aproximadamente 3 segundos y es el momento crítico de la prueba ya que es durante ésta cuando se lleva a cabo la medición de la $\operatorname{CRF}\left(\mathrm{V}_{1}\right)$. Una vez abierta la válvula, el paciente debe realizar una inspiración máxima (para la medición de la $\mathrm{Cl}$ ) y, posteriormente, una exhalación completa de manera lenta y relajada (Figura 3). La CPT es la suma de la CRF más la $\mathrm{Cl}$.

Las principales mediciones de la pletismografía corporal por su utilidad clínica son la CPT, el VR y la $\mathrm{Cl}$; aunque esta prueba también permite medir otros volúmenes, como el VC, el de reserva inspiratoria y espiratoria, así como las resistencias pulmonares.

La CPT es el máximo volumen de aire (medido en $\mathrm{L}$ ) que puede ser contenido por los pulmones, lo que se traduce en tamaño pulmonar. Con esta medición podemos hacer el diagnóstico definitivo de restricción, así como de hiperinflación pulmonar, para este último diagnóstico también es necesario considerar al VR. El VR es la cantidad de aire que se mantiene en los pulmones después de una espiración máxima y nos indica si existe atrapamiento de aire.

La principal indicación para realizar una pletismografía corporal es la sospecha de enfermedad restrictiva del tórax. La sospecha de un proceso restrictivo suele fundamentarse, desde el punto de vista fisiológico, en la disminución de la CVF obtenida en la espirometría y se confirma con la disminución de la CPT a través de la pletismografía. ${ }^{2}$ Las enfermedades restrictivas intrapulmonares, clásicamente la enfermedad pulmonar parenquimatosa difusa, generan disminución de todos los volúmenes. Por el contrario, cuando la restricción pulmonar se debe a enfermedad neuromuscular puede documentarse disminución de la CPT con aumento del VR, lo que incrementa la relación VR/CPT. No sólo los procesos obstructivos aumentan dicho cociente. ${ }^{4}$

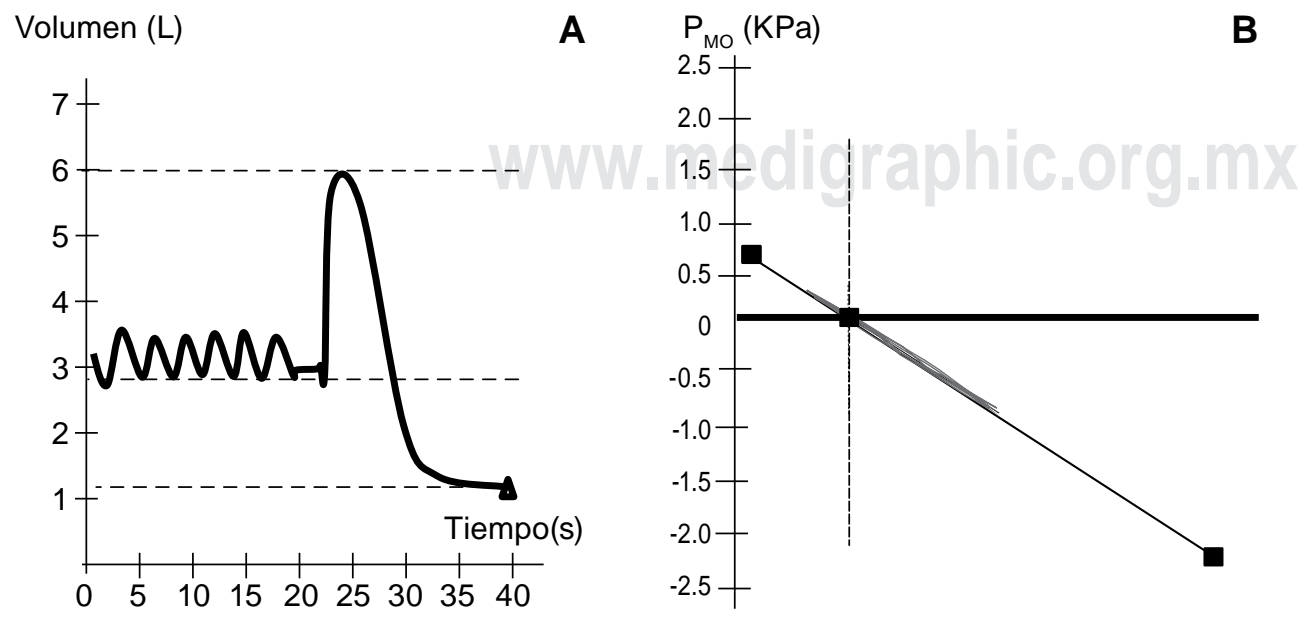

Figura 3:

Pletismografía corporal. El panel A muestra la gráfica volumen-tiempo donde debe obtenerse volumen corriente estable, posteriormente se produce la oclusión del obturador por 3-5 segundos (línea recta pequeña); después se solicita al paciente que realice una inspiración profunda seguida de una espiración completa de manera relajada. El panel B muestra la curva obtenida durante la oclusión. Debe tener histéresis mínima y deben observarse ambos extremos de la curva (criterios de aceptabilidad). $\mathrm{P}_{\mathrm{MO}}$ : Presión en la boca. 
La CPT incrementada es un marcador funcional de hiperinflación pulmonar que acompaña a los procesos obstructivos graves. Otro parámetro que indica hiperinflación es el cociente $\mathrm{Cl} / \mathrm{CPT}$, éste tiene implicaciones pronosticas en EPOC. ${ }^{10}$ Las contraindicaciones de la prueba son las mismas que para la espirometría (ver sección de espirometría arriba). Si bien, la prueba es muy segura existen complicaciones potenciales que son compartidas por la espirometría y que son: síncope, accesos de tos, broncoespasmo, dolor torácico, aumento de presión intracraneal y crisis de ansiedad.

Al igual que con la espirometría deberán obtenerse tres maniobras aceptables. Para la repetibilidad, la variable tomada en cuenta es la capacidad vital (CV). La diferencia entre las dos mejores maniobras deberá ser $<150 \mathrm{~mL}$; sin embargo, en la pletismografía también se evalúa la varianza del VGIT, que no debe rebasar el $5 \%{ }^{9}$ Aunque no se han establecido criterios claros para la evaluación de los patrones funcionales obtenidos por pletismografía corporal, nosotros proponemos los valores que se muestran en la Tabla 3.

\section{DIFUSIÓN PULMONAR DE MONÓXIDO DE CARBONO CON RESPIRACIÓN ÚNICA}

La difusión pulmonar de monóxido de carbono $\left(\mathrm{DL}_{\mathrm{co}}\right)$ es una prueba de intercambio gaseoso que permite evaluar el proceso de transferencia de oxígeno, desde el gas alveolar hasta su unión con la hemoglobina contendida en los glóbulos rojos. ${ }^{11}$ Para tal fin, el oxígeno debe cruzar la pared alveolar, la pared del capilar, el plasma, la membrana del eritrocito y finalmente unirse a la hemoglobina. La cantidad de oxígeno que puede ser transferido del gas alveolar hasta su unión con la hemoglobina está determinado por tres factores principales: el área de la membrana alveolocapilar; el grosor de la misma, y el gradiente de presión de oxígeno entre el gas alveolar y la sangre venosa. La medición de la capacidad de $\mathrm{DL}_{\mathrm{co}}$ es simple y es una estimación válida de la capacidad de difusión de oxígeno. Así, la ecuación de $\mathrm{DL}_{\mathrm{CO}}$ podría ser expresada como $\mathrm{DL}_{\mathrm{CO}}=\mathrm{A} \times \Delta \mathrm{PCO} / \mathrm{G}$, donde $\mathrm{DL}_{\mathrm{CO}}$ es la capacidad de difusión de monóxido de carbono, A es el área, $\triangle \mathrm{PCO}$ es la diferencia de presiones de monóxido de carbono entre el gas alveolar y la sangre venosa y $\mathrm{G}$ es el grosor de la membrana alveolocapilar.

Al igual que para otras PFR, la utilidad de esta prueba es muy amplia pues sirve como prueba complementaria de diagnóstico para dar seguimiento y evaluar la respuesta terapéutica. Esta prueba, junto con una de mecánica pulmonar (espirometría simple o con broncodilatador) y con una dinámica (C6M), son las pruebas iniciales en la evaluación de la mayoría de los pacientes con enfermedad respiratoria.

Cualquier paciente en quien sea necesario evaluar el intercambio de gases en estado de reposo, es candidato a realizar esta prueba; pero es más relevante en las enfermedades que suelen afectar inicialmente el intercambio y no la mecánica pulmonar tales como las enfermedades intersticiales de pulmón o enfermedades de la circulación pulmonar. Aunque no existen contraindicaciones absolutas para su realización, los sujetos que utilizan oxígeno suple-

Tabla 3: Patrones funcionales propuestos y obtenidos a partir de la pletismografía corporal.

\begin{tabular}{|c|c|l|}
\hline Parámetro & Porcentaje del predicho & Gravedad \\
\hline CPT & $>120$ & Obstrucción con hiperinflación \\
& $80-119$ & Normal \\
& $70-79$ & Restricción leve \\
& $60-69$ & Restricción moderada \\
& $50-59$ & Restricción moderadamente grave \\
& $35-49$ & Restricción grave \\
& $<35$ & Restricción muy grave \\
\hline CV & $80-119$ & Normal \\
& $70-79$ & Restricción leve \\
& $60-69$ & Restricción moderada \\
& $50-59$ & Restricción moderadamente grave \\
& $35-49$ & Restricción grave \\
& $<35$ & Restricción muy grave \\
\hline VR & $<119$ & Normal \\
& $>120$ & Atrapamiento aéreo leve \\
& $131-139$ & Atrapamiento aéreo moderado \\
& $>140$ & Atrapamiento aéreo grave \\
\hline
\end{tabular}


Volumen (L)

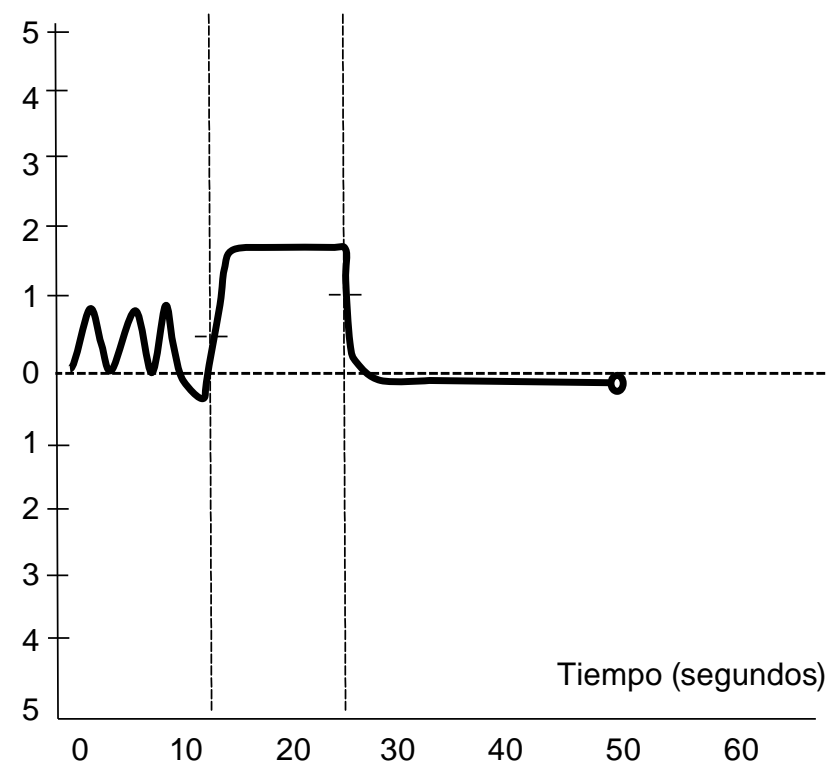

Figura 4: Prueba de difusión de monóxido de carbono. La prueba consiste en hacer una serie de respiraciones a volumen corriente, luego una espiración completa y después una inspiración máxima de la mezcla de gases, seguida de una apnea de $10 \pm 2$ segundos (con el fin de que el $\mathrm{CO}$ se distribuya de manera homogénea en los alvéolos); y para finalizar la prueba, una exhalación con una maniobra relajada. Las líneas pequeñas en la inspiración y espiración muestran el momento en que se lleva a cabo la medición de la cantidad de $\mathrm{CO}$ entregado (por el equipo) y el recuperado en la exhalación. CO: Monóxido de carbono.

mentario no son buenos candidatos ya que es necesario suspender el oxígeno por lo menos 5 minutos antes de la prueba, lo cual no siempre es posible.

Existen varios métodos para medir la tasa de transferencia de monóxido de carbono $(\mathrm{CO})$ o $\mathrm{DL}_{\mathrm{CO}}$. La técnica de respiración única $\left(\mathrm{DL}_{\mathrm{CO}} \mathrm{sb}\right)$ es la más utilizada. ${ }^{11}$ Se requiere de un equipo especializado que hasta hace algunos años tenía la desventaja de ser poco portátil y prácticamente exclusivo de los laboratorios de función pulmonar. Sin embargo, recién se ha desarrollado tecnología que permite que este dispositivo sea de gran portabilidad y su manejo sumamente amigable.

Para llevar a cabo la prueba de $\mathrm{DL}_{\mathrm{CO}}$ sb se utiliza una mezcla especial de gases (CO al $0.03 \%$, helio al $8 \%$, oxígeno al $21 \%$ y nitrógeno para balancear la mezcla). El resultado es expresado en $\mathrm{mL}$ de $\mathrm{CO} / \mathrm{min} / \mathrm{mmHg}$.

La maniobra que el enfermo debe realizar consiste en respiraciones a VC estable seguido de una espiración hasta VR, posteriormente, la realización de una inspiración completa. Una vez que el técnico está seguro de haber conseguido ésta, le pide al sujeto que haga una apnea de 10 segundos (tiempo durante el cual se distribuye y homogeniza la mezcla de gases en los espacios alveolares), y que finalmente lleve a cabo, otra vez, una espiración completa (Figura 4). Es al inicio de la maniobra espiratoria que el analizador de gases realiza las mediciones de las concentraciones de CO exhalado. Deberán obtenerse al menos dos maniobras aceptables y repetibles entre los mejores valores de $\mathrm{DL}_{\mathrm{CO}}$. Se permite un máximo de cuatro maniobras con un tiempo mínimo entre cada una de ellas de 4 minutos. Para considerar una prueba repetible es necesario que las mediciones de $\mathrm{DL}_{\mathrm{CO}}$ no difieran en más de tres unidades. Los resultados de $\mathrm{DL}_{\mathrm{CO}}$ obtenidos deberán ajustarse por la altitud del lugar en el que se realizó la prueba (en la Ciudad de México el factor de corrección es de 0.87), así como por la concentración de hemoglobina. Para la interpretación de la prueba es necesario obtener el promedio de dos maniobras aceptables y repetibles. Desde un punto de vista práctico, la $\mathrm{DL}_{\mathrm{CO}}$ disminuye en enfermedades que afectan a alguno de los componentes de la ecuación $\left(\mathrm{DL}_{\mathrm{CO}}=\mathrm{Ax}\right.$ $\triangle \mathrm{PCO} / \mathrm{G}$; ver arriba); p. ej., si disminuye $\mathrm{A}$ como sucede en el enfisema o en pacientes con resecciones pulmonares, disminuye la $\mathrm{DL}_{\mathrm{CO}}$; de manera inversa, si se incrementa $\mathrm{G}$ como sucede en las enfermedades del intersticio pulmonar, disminuye la $\mathrm{DL}_{\mathrm{co}}$. En diversas enfermedades, especialmente las de tipo intersticial, las alteraciones en el intercambio de gases (en este caso evaluado mediante $\left.\mathrm{DL}_{\mathrm{CO}}\right)$ preceden a las alteraciones en la mecánica de la respiración (espirometría o pletismografía). La Tabla 4 muestra los grados de gravedad de disminución de $\mathrm{DL}_{\mathrm{CO}}$. $\mathrm{La} \mathrm{DL}_{\mathrm{CO}}$ también puede estar incrementada, lo anterior se observa en pacientes con hemorragia pulmonar o asma.

\section{CAMINATA DE SEIS MINUTOS}

La C6M es una prueba de ejercicio que mide la distancia que un individuo puede caminar, tan rápido como le sea posible, en una superficie dura y plana (usualmente en un corredor de $30 \mathrm{~m}$ ) durante un período de seis minutos. ${ }^{12,13}$ La mayor utilidad práctica de esta prueba es analizar los efectos del tratamiento sobre la capacidad de ejercicio (metros caminados). Además, permite medir el estatus funcional de los pacientes con diversas enfermedades, en algunas de ellas es un predictor de mortalidad. ${ }^{14-16}$ Aunque

Tabla 4: Grados de gravedad de la disminución en la capacidad de difusión de monóxido de carbono $\left(\mathrm{DL}_{\mathrm{co}}\right)$.

\begin{tabular}{|l|c|}
\hline $\begin{array}{l}\text { Gravedad } \\
\mathrm{DL}_{\mathrm{co}} \% \text { predicho }\end{array}$ \\
\hline Leve & $>60 \% \mathrm{y}<\mathrm{LIN}$ \\
\hline Moderado & $40 \mathrm{a} 60 \%$ \\
\hline Grave & $<40 \%$ \\
\hline
\end{tabular}

LIN: Límite inferior de la normalidad. 
el principal parámetro medido son los metros caminados, existen otros aspectos funcionales que también se pueden analizar como es, el estado de oxigenación (oximetría de pulso) o la disnea que presenta el paciente durante el desarrollo de la prueba. ${ }^{17}$

La C6M tiene requerimientos técnicos que están ampliamente disponibles pues sólo es necesario un corredor (en interiores) de $30 \mathrm{~m}$ (idealmente no transitado y con conos de tráfico), un cronómetro y personal capacitado en la realización de la prueba. Según los estándares de la Sociedad Americana del Tórax (ATS, por sus siglas en inglés),$^{12}$ no es obligada la medición de la saturación de oxígeno durante la prueba; sin embargo, nosotros la recomendamos ampliamente; de realizarse, lo ideal es que sea con un oxímetro de pulso ligero y diseñado para minimizar artefactos de movimiento. Aun cuando no es una recomendación rutinaria, es aconsejable realizar la C6M en dos ocasiones (con al menos 30 minutos de intervalo entre ellas o hasta que los signos vitales retornen a sus valores basales) y tomar la prueba en la que se caminaron más metros (debido al efecto de aprendizaje).

Cualquier individuo que pueda caminar puede realizar una C6M; aunque su utilidad ha sido estudiada y demostrada sobre todo en pacientes con EPOC. El ejemplo más relevante quizá sea la incorporación de la C6M en el índice multidimensional BODE (Body Mass Index, Obstruction, Dyspnea, Exercise Capacity) utilizado en la evaluación de pacientes con EPOC. ${ }^{14}$ Este índice ha demostrado predicir la mortalidad en pacientes con esta enfermedad y, más aún, un estudio interesante publicado por Martínez et ál. ${ }^{18}$ demostró que la disminución de un punto en dicha escala (la escala es de 0 a 10 puntos) disminuye la mortalidad. La manera más eficiente para disminuir el índice BODE es incrementando los metros que un sujeto puede caminar en seis minutos mediante la implementación de estrategias encaminadas a disminuir la hiperinflación dinámica y con programas de rehabilitación respiratoria. ${ }^{19}$ La PCPE no es superior a la C6M en términos de la habilidad predictiva del índice BODE. ${ }^{20}$ Lo mencionado es tan sólo un ejemplo de la utilidad de C6M, la cual debe de ser realizada en todos los pacientes portadores de EPOC. En otras enfermedades, la C6M también ha mostrado ser de utilidad, tal es el caso de la hipertensión arterial pulmonar, la fibrosis pulmonar, la fibrosis quística e incluso, en enfermedades cardiovasculares como la insuficiencia cardíaca. ${ }^{13,15,16,18,21,22}$ Consideramos que el neumólogo actual debe realizar la C6M con la misma familiaridad y frecuencia con la que realiza una espirometría. Un aspecto importante es que el uso de auxiliares ortopédicos no contraindica que se lleve a cabo la prueba.

Una de las limitaciones que tiene la C6M es que no existen valores de referencia que puedan ser utilizados ampliamente. Esto obliga a utilizar el número absoluto de metros caminados en lugar del porcentaje del esperado. Al igual que para otras PFR, debemos contar con valores de referencia locales. Algunos estudios han demostrado que los valores de referencia disponibles no ajustan apropiadamente a lo caminado por sujetos sanos en poblaciones diferentes de la que dio origen a los valores de referencia. ${ }^{23}$ Existen aspectos culturales, más allá de los aspectos antropomórficos, que afectan lo que los individuos sanos caminan; por ejemplo, es posible que en Europa se camine más que en Estados Unidos. Estas diferencias culturales e incluso, las diferencias en composición corporal, afectan los resultados de estudios que aspiran a generar valores de referencia. En México, Luna et ál. publicaron valores de referencia. ${ }^{24}$ En tanto no se disponga de valores de referencia apropiados, la mejor utilidad de la prueba es en el análisis longitudinal de la medición; es decir, analizar y cuantificar el cambio (en metros caminados) que tiene un individuo en relación al tiempo y a los tratamientos instituidos. Otro aspecto que merece un comentario con relación a la $\mathrm{C} 6 \mathrm{M}$ es que no se ha definido con precisión cuál es la diferencia en metros después de una intervención dada que es clínicamente significativa. Se ha propuesto un punto de corte de entre 54 metros para los pacientes con enfermedades obstructivas ${ }^{24}$ y 24 a 45 metros en el caso de enfermedades intersticiales, aunque se requieren más estudios que permitan dilucidar este punto. ${ }^{22,25}$

Existen algunas contraindicaciones para realizar la C6M, como angina inestable y/o infarto miocárdico en el mes previo y limitación física que imposibilite deambular. Las contraindicaciones relativas incluyen: presión arterial sistólica $\geq 180 \mathrm{mmHg}$, presión arterial diastólica $\geq 100$ mmHg y frecuencia cardiaca $\geq 120$ latidos por minuto.

El informe de la C6M se origina a partir de aquella en la que se hayan caminado más metros (ver arriba) y debe incluir los datos demográficos, las mediciones antropométricas (realizadas en forma estandarizada), los datos para identificar al técnico y el diagnóstico presuntivo o definitivo, además, los signos vitales, la escala de disnea de Borg y, cuando está disponible, la saturación de oxígeno por oximetría de pulso $\left(\mathrm{SpO}_{2}\right)$ basal y postprueba. Debe registrarse si el paciente interrumpió la prueba y los motivos para ello. Las mediciones realizadas al minuto 1 y 5 posterior a haber terminado la prueba podrían ser de utilidad clínica. ${ }^{26}$ Asimismo, como un recurso de control de calidad, es aconsejable incluir el porcentaje de la frecuencia cardiaca máxima alcanzado durante la prueba.

\section{PRUEBA DE RETO CON EJERCICIO}

Es una prueba de mecánica respiratoria que permite evaluar la respuesta bronquial al ejercicio. En condiciones normales, el ejercicio no provoca obstrucción bronquial; pero, la mayoría de los pacientes con asma desarrollan 
bronconstricción cuando realizan esta prueba. ${ }^{6}$ El principio que la sustenta es que el aumento del volumen minuto secundario al incremento en las demandas metabólicas induce pérdida de agua en el epitelio bronquial, lo que seca y enfría el aire que conforma el VC. El aire seco y frío estimula la liberación de histamina y leucotrienos, ambas son sustancias involucradas en la obstrucción bronquial. ${ }^{6}$

La prueba puede realizarse con diferentes protocolos de ejercicio, los más comunes son el uso de banda sin fin (que permite aumentar progresivamente la velocidad y la inclinación) o en una bicicleta (cuya resistencia es controlada de manera electrónica). Se realiza bajo condiciones ambientales estandarizadas a una temperatura entre 20 y $25{ }^{\circ} \mathrm{C}$ y una humedad relativa menor al $50 \%$. El paciente debe realizar la prueba respirando por la boca y utilizando pinzas nasales (la respiración nasal disminuye la pérdida de agua y calienta el aire inspirado). La temperatura y la humedad relativa deben ser registradas. La duración máxima de la prueba es de 8 minutos. La prueba inicia a baja velocidad e inclinación. Hay que realizar una espirometría antes de realizar el ejercicio (espirometría basal) y, una vez concluido éste, una cada 5 minutos hasta los 20 minutos (comenzando en el minuto 1 después del ejercicio). Las espirometrías deben realizarse siguiendo los lineamientos establecidos por la ATS (por lo menos tres esfuerzos aceptables por cada espirometría y con repetibilidad menor a $200 \mathrm{~mL}$ en $\mathrm{VEF}_{1}$ entre las pruebas). ${ }^{7}$ En las espirometrías postejercicio, el tiempo de exhalación puede reducirse a 3 segundos pues el resultado más importante es el $\mathrm{VEF}_{1}$. Debe administrarse broncodilatador de acción corta (agonista $\beta_{2}$ adrenérgico) en caso de que el enfermo presente disnea grave o cuando la prueba sea positiva o haya evidencia clara de broncoconstricción.

La prueba de reto con ejercicio está indicada en pacientes con historia sugestiva de asma pero sólo en quienes no se ha logrado demostrar en reposo la obstrucción al flujo aéreo, pacientes que presentan sintomatología atípica o que únicamente manifiestan un síntoma (tos crónica, opresión torácica, disnea de esfuerzo) o en sujetos que presentan sintomatología sugestiva de hiperreactividad bronquial únicamente al realizar ejercicio (asma inducida por ejercicio), así como en los casos en los que esté contraindicada la prueba de reto de metacolina, o bien ésta sea negativa. ${ }^{6,7}$

La prueba está contraindicada de manera absoluta cuando la limitación al flujo aéreo es grave $\left(\mathrm{VEF}_{1}<50 \%\right.$ del predicho o $<1 \mathrm{~L}$ ), así como en sujetos que han sufrido un infarto agudo al miocardio en los últimos tres meses, hipertensión arterial descontrolada (presión arterial sistólica $>200 \mathrm{mmHg}$ o diastólica > $100 \mathrm{mmHg}$ ) o si hay conocimiento de un aneurisma. Existe poca justificación para realizar esta prueba en quienes ya se demostró la obstrucción bronquial mediante pruebas rutinarias. Hay también contraindicaciones relativas que incluyen limitación moderada al flujo aéreo $\left(\mathrm{VEF}_{1}<60 \%\right.$ del predicho o $\left.<1.5 \mathrm{~L}\right)$, embarazo, lactancia, uso de medicamentos análogos a la colinesterasa, isquemia cardiaca, angina inestable, arritmias y limitaciones ortopédicas.

La prueba es muy sencilla de interpretar. Aunque aún existe debate del punto de corte para considerar una prueba positiva, nosotros recomendamos interpretarla como positiva cuando el $\mathrm{VEF}_{1}$ disminuya $\geq 10 \%$ con respecto a la espirometría realizada antes del ejercicio; aunque se hace más específica si se considera una caída del $15 \%$ en el $\mathrm{VEF}_{1}{ }^{6}$

\section{PRUEBA DE DESATURACIÓN Y DE TITULACIÓN DE OXÍGENO SUPLEMENTARIO}

La prueba de desaturación de oxígeno (PDO) y la de titulación de oxígeno suplementario (PTOS) son pruebas complementarias de intercambio gaseoso. La primera evalúa el grado de hipoxemia que se presenta durante el ejercicio (en un período de 6 minutos) y la segunda evalúa el efecto del oxígeno suplementario sobre la saturación de oxígeno. ${ }^{27}$ El principio de la PDO radica en que en individuos sanos al realizar ejercicio, la presión parcial de oxígeno en sangre arterial $\left(\mathrm{PaO}_{2}\right)$ y la saturación de oxígeno $\left(\mathrm{SaO}_{2}\right)$ se mantienen sin cambios o incrementan, mientras que en pacientes con enfermedades pulmonares o cardiovasculares la oxigenación disminuye.

Existen varios protocolos de ejercicio que pueden ser utilizados durante la PDO y la PTOS. Lo usual es que el ejercicio realizado durante la PDO sea submáximo; es decir, que el sujeto realice un esfuerzo que en magnitud semeje al requerido para llevar a cabo las actividades de la vida diaria. Existen varias pruebas de ejercicio submáximo, las más frecuentes incluyen protocolos llevados a cabo mediante caminata en banda sin fin o caminata en corredor. La decisión del protocolo de ejercicio que se llevará a cabo descansará sobre la disponibilidad de equipo y del estado físico del paciente. ${ }^{27}$

La PDO tiene su principal utilidad al demostrar hipoxemia antes de que las pruebas de intercambio gaseoso realizadas en condiciones de reposo muestren alguna alteración. La hipoxemia que sólo se documenta en el ejercicio suele ser expresión temprana de enfermedad.

La utilidad de la PTOS radica en permitir identificar el flujo apropiado de oxígeno suplementario que el paciente necesita ya sea en reposo o ejercicio para mantener un umbral dado de oxigenación, p. ej., $\mathrm{SpO}_{2} \geq 90 \%$.

La PDO está indicada en pacientes que cursan con síntomas respiratorios (usualmente disnea) en quienes las pruebas en reposo no documentan alteraciones en el intercambio de gases. El ejemplo clásico son los pacientes con enfermedades pulmonares parenquimatosas difusas 
quienes en las etapas iniciales manifiestan disnea sólo durante el ejercicio. ${ }^{28}$ También se puede realizar PDO para evaluar la respuesta al tratamiento; sin embargo, para tal fin recomendamos de preferencia la C6M o la prueba de ejercicio cardiopulmonar.

La PTOS está indicada en pacientes con hipoxemia (en reposo y/o ejercicio). La PDO no debe realizarse en pacientes que presentan hipoxemia en reposo debido a que la prueba tiene como objetivo documentar si el ejercicio submáximo induce o no desaturación de oxígeno; luego entonces, resulta innecesario realizar la prueba en quien se encuentra hipoxémico en reposo. Igualmente obvio resulta decir que la normooxemia contraindica realizar una PTOS.

La interpretación de la PDO es simple y consiste en definir si la prueba es positiva. Los criterios para considerar la prueba como positiva podrían ser diferentes dependiendo de la altitud sobre el nivel del mar. No obstante, proponemos que la PDO sea considerada positiva (en la Ciudad de México) cuando en el transcurso de 6 minutos se presente cualquiera de lo siguiente: ${ }^{27}$

a. $\mathrm{SpO}_{2} \leq 88 \%$ de manera sostenida por al menos un minuto;

b. $\mathrm{SpO}_{2} \leq 85 \%$ por 15 segundos;

c. disminución al menos del $4 \%$ de la $\mathrm{SpO}_{2}$ basal por al menos 1 minuto (aunque la $\mathrm{SpO}_{2}$ no esté por debajo de $88 \%$ ).

Cuando la PDO es realizada en banda sin fin y existe un catéter arterial, debe incluirse el siguiente criterio gasométrico:

a. disminución de $\mathrm{PaO}_{2} \geq 5 \mathrm{mmHg}$ con respecto al valor basal y/o;

b. disminución de $\mathrm{SaO}_{2} \geq 3 \%$ con respecto al valor basal;

c. $\mathrm{PaO}_{2}$ al final de la prueba $\leq 55 \mathrm{mmHg}$;

d. $\mathrm{SaO}_{2}$ al final de la prueba $\leq 88 \%$.

Algunos pacientes pueden reunir criterios de prueba positiva de acuerdo con los parámetros gasométricos y ser negativos al considerar la $\mathrm{SpO}_{2}$ (criterio de oximetría). En esos casos es recomendable dar preferencia a los valores obtenidos de la gasometría dado que es el estándar de referencia.

\section{PRUEBA CARDIOPULMONAR DE EJERCICIO}

La PCPE permite evaluar de manera simultánea la capacidad de los sistemas respiratorio y cardiovascular para realizar el intercambio gaseoso entre las células y el medio ambiente en circunstancias de alta demanda metabólica. ${ }^{29}$ Durante la PCPE el paciente realiza su máximo esfuerzo para realizar ejercicio. Usualmente la PCPE se lleva a cabo utilizando una bicicleta (cicloergómetro) cuya resistencia al pedaleo es electrónicamente controlada. Cuando se trata de un protocolo incremental -que es lo más frecuente- la resistencia se aumenta de manera progresiva y la prueba es limitada por síntomas; es decir, el paciente decide en qué momento interrumpe la prueba debido a la presencia de disnea o fatiga en las piernas. En algunas ocasiones existen otras causas por las que se detiene la prueba e incluyen: dolor precordial, mareo, trastornos del ritmo cardiaco o de la conducción, o cualquier otra circunstancia que a juicio del médico requiera que la prueba sea suspendida aun y cuando el paciente considere que puede continuar. ${ }^{30}$

A diferencia del protocolo incremental, la PCPE también puede ser realizada en protocolo de carga constante. ${ }^{30}$ Este protocolo solicita al paciente que se ejercite cuanto le sea posible a una misma intensidad de trabajo, la cual es preestablecida mediante una prueba incremental, usualmente entre el 70 y $80 \%$ de su capacidad máxima. La prueba de carga constante es particularmente útil porque toma en cuenta el tiempo que el paciente resiste realizando ejercicio a una misma carga de trabajo. Lo anterior permite evaluar la respuesta a los tratamientos farmacológicos y a las estrategias de rehabilitación. Mientras mejor sea la respuesta a los tratamientos instituidos, mayor será el tiempo que el paciente logra hacer ejercicio a una carga constante de trabajo. ${ }^{30}$

La PCPE es de utilidad ya que permite: 1) analizar de manera integral la respuesta al ejercicio; 2) medir objetivamente la capacidad de ejercicio; 3) evaluar la reserva funcional de los sistemas implicados en el ejercicio; 4) identificar los mecanismos que limitan la tolerancia al ejercicio; 5) conocer el grado de limitación al ejercicio con fines de dictaminar incapacidad o impedimento; 6) establecer índices pronósticos (incluyendo riesgo operatorio); 7) planear programas de rehabilitación respiratoria, y 8) evaluar la progresión de la enfermedad y la respuesta a diversas intervenciones terapéuticas. ${ }^{31}$

La indicación clásica de la PCPE es el estudio de pacientes con disnea en quienes después de realizar estudios en condiciones de reposo, no se ha logrado identificar la causa de dicho síntoma. Esta indicación se sustenta en que la PCPE permite establecer patrones de limitación al ejercicio, limitación respiratoria o limitación cardiovascular. Por lo anterior, la PCPE es de utilidad en el diagnóstico diferencial de enfermedades cardiopulmonares. ${ }^{31}$

Existen contraindicaciones absolutas para realizar una PCPE que incluyen: infarto agudo del miocardio en los ocho días previos, angina inestable, arritmias cardiacas no controladas, endocarditis infecciosa activa, miocarditis o pericarditis aguda, estenosis aórtica grave, insuficiencia cardiaca no controlada, tromboembolia pulmonar aguda, enfermedades que puedan ser agravadas por el ejercicio 


\section{GASOMETRÍA ARTERIAL Y OXIMETRÍA DE PULSO} agudas graves, etc.) y trombosis venosa en extremidades inferiores. Las contraindicaciones relativas -en las que debe valorarse si el beneficio es mayor que el riesgo - son: estenosis de la arteria coronaria izquierda, estenosis valvular cardiaca moderada, alteraciones electrolíticas, hipertensión arterial sistémica no tratada, hipertensión pulmonar grave, cardiomiopatía hipertrófica, limitaciones mentales y bloqueo auriculoventricular.

Los principales parámetros obtenidos de la PCPE son el consumo de oxígeno $\left(\mathrm{VO}_{2}\right)$ y la producción de bióxido de carbono $\left(\mathrm{VCO}_{2}\right)$. Estas mediciones se obtienen en cada respiración mediante un analizador de gases previamente calibrado. La comparación del $\mathrm{VO}_{2}$ medido contra los valores de referencia permite dilucidar si el paciente presenta baja capacidad de ejercicio. La disminución en la capacidad de ejercicio puede tener diversos orígenes, siendo precisamente este punto en el cual la PCPE aporta su mayor utilidad clínica. ${ }^{31}$

Como ya se mencionó, durante la PCPE se miden diversos parámetros, tanto cardiovasculares como respiratorios que permiten dilucidar si la disminución en la capacidad de ejercicio es debida a limitación cardiovascular o a limitación respiratoria. Las definiciones de ambas limitaciones son relativamente arbitrarias; p. ej., los pacientes portadores de EPOC o de otras enfermedades respiratorias crónicas agotan su reserva respiratoria, es decir, en el momento que realizan su máxima capacidad de ejercicio tienen una ventilación minuto que alcanza, o al menos es muy cercana ( $\geq 85 \%$ ), a su máxima capacidad de ventilar (ventilación voluntaria máxima [VVM]). Esto contrasta con lo que sucede en condiciones normales (o no respiratorias) en donde aún en el pico de ejercicio, el individuo mantiene una reserva respiratoria de aproximadamente 15\% de su VVM. Los atletas de alto rendimiento, a diferencia de los sujetos normales no entrenados, sí pueden agotar su reserva respiratoria. Otros parámetros relacionados a la limitación respiratoria son la disminución progresiva de la capacidad inspiratoria durante el ejercicio (debido a hiperinflación dinámica), disminución de la oxigenación y elevación de la $\mathrm{PaCO}_{2}$. Por el contrario, los pacientes con limitación cardiovascular tienen un umbral láctico temprano, alcanzan rápidamente su frecuencia cardiaca máxima esperada, cursan con bajo pulso de oxígeno (que es el cociente $\mathrm{VO}_{2}$ /frecuencia cardiaca) y mantienen gran reserva respiratoria. La interpretación detallada de la PCPE escapa a los objetivos de este escrito. Para tal fin recomendamos la revisión de otros documentos. 2,3,30,31

La medición de la capacidad de ejercicio también es de utilidad para valorar el riesgo operatorio. Existen puntos de corte del consumo máximo de oxígeno $\left(\mathrm{VO}_{2}\right.$ max $)$ que han demostrado ser predictores confiables de mortalidad operatoria, y a su vez son utilizados con fines de dictaminar incapacidad. ${ }^{31}$
La gasometría arterial (GA) es una prueba que permite analizar de manera simultánea varios aspectos fisiológicos que incluyen: la ventilación alveolar, el estado ácido base y el estado de oxigenación. El equilibrio entre estos tres factores, los cuales se encuentran estrechamente relacionados, depende de la respuesta integrada de varios sistemas que abarcan al aparato respiratorio, cardiovascular, hematológico y renal, entre otros. Aunque la GA evalúa aspectos más amplios que la mera función pulmonar, la incluimos en este documento debido a que es una prueba de utilidad cotidiana en la práctica neumológica que tiene implicaciones diagnósticas, terapéuticas y pronosticas. Además, al igual que otras PFR, la GA permite cuantificar el efecto de las intervenciones (médicas, ventilatorias o quirúrgicas) sobre la evolución de las enfermedades (agudas o crónicas) que afectan al aparato respiratorio. La utilidad práctica de la GA en la atención de pacientes mecánicamente ventilados es incuestionable y la GA es indispensable para establecer el diagnóstico de insuficiencia respiratoria. Con lo mencionado hasta aquí, se desprende que la GA tiene aplicación en casi cualquier rama de la medicina y las indicaciones para su realización son múltiples.

Existen algunas contraindicaciones para realizar GA, entre ellas: circulación colateral inadecuada (usualmente en el territorio de la arteria radial), lesión en el sitio de punción, presencia de cortocircuito quirúrgico en la extremidad a puncionar, anticoagulación excesiva, administración de trombolíticos o en pacientes con defectos congénitos de la coagulación (algunas contraindicaciones son relativas). Como cualquier otro procedimiento invasivo, la GA no está exenta de complicaciones como: espasmo arterial, reacción vagal, hematomas, hemorragia, trombosis y lesión vascular o neural, etc. ${ }^{32}$

De manera sucinta plantearemos algunas recomendaciones generales acerca de la interpretación de la GA. Es importante conocer las condiciones clínicas en las que fue obtenida la muestra; es decir, se debe anotar el diagnóstico del paciente y si éste se encontraba en reposo, en ejercicio o bajo ventilación mecánica y si así es, bajo qué parámetros del ventilador. Otro aspecto necesario para la correcta interpretación de la GA es conocer los valores normales considerando la altitud sobre el nivel del mar, pues de lo contrario podrían cometerse errores de interpretación no sólo en el aspecto de intercambio gaseoso, sino también en el ácido base. ${ }^{33,34}$

El estado ventilatorio se evalúa mediante la $\mathrm{PaCO}_{2}$ al compararla contra los valores de referencia para la altitud. En general, se puede estimar la $\mathrm{PaCO}_{2}$ normal para un lugar dado si se conoce su altitud sobre el nivel del mar utilizando la siguiente fórmula: $\mathrm{PaCO}_{2}=40-(3.96 \mathrm{x}$ 
altitud en km). Si la $\mathrm{PaCO}_{2}$ se encuentra por arriba de los valores de referencia refleja disminución en la ventilación alveolar. Recordemos que la $\mathrm{PaCO}_{2}$ está en función inversa a la ventilación alveolar $\left(\mathrm{V}_{\mathrm{A}}\right)$ y en función directa a la producción de $\mathrm{CO}_{2}\left(\mathrm{VCO}_{2}\right)$; es decir, $\mathrm{PaCO}_{2}=\mathrm{VCO}_{2} \times \mathrm{k} /$ $\mathrm{V}_{\mathrm{A}}$. Las causas que originan hipoventilación son múltiples e incluyen hipoventilación central por drogas o enfermedades del sistema nervioso central (isquemia, tumores, etc.) e hipoventilación asociada a enfermedades restrictivas extrapulmonares, tales como la obesidad mórbida, defectos de la caja torácica que limitan la expansión pulmonar o enfermedades neuromusculares.

Cuando la $\mathrm{PaCO}_{2}$ disminuye por abajo de su valor de referencia reflejará un incremento en la ventilación alveolar. Existen varias causas de hipocapnia que incluyen: dolor (incluso al tomar la muestra de sangre arterial para realizar la GA), trastornos del control central de la respiración (enfermedad cerebrovascular o trauma craneoencefálico), estados asociados a ansiedad o trastornos conversivos y estados que cursan con acidosis metabólica aguda (cetoacidosis diabética, insuficiencia renal aguda o intoxicación por salicilatos), entre otros. La exacerbación leve o moderada de asma, así como la tromboembolia pulmonar suelen cursar con hipocapnia dado que existe incremento en el volumen minuto como consecuencia de la hipoxemia originada por el desequilibrio entre la ventilación y la perfusión $\left(\mathrm{V}_{\mathrm{A}} / \mathrm{Q}\right)$. La hipocapnia es frecuente en pacientes con ventilación mecánica.

De acuerdo a lo ya comentado, la GA es de gran utilidad en la evaluación del estado ácido-base. Pocos sistemas en el organismo requieren de un control tan riguroso como el equilibrio ácido-base, lo que puede constatarse por el estrecho margen de normalidad del pH (de 7.35 a 7.45). No es el propósito de este documento agotar el tema de la interpretación del estado ácido-base, sólo haremos hincapié en que debe ser evaluado considerando la altitud sobre el nivel del mar. ${ }^{33}$ La altitud disminuye los valores de $\mathrm{PaCO}_{2}$ y de bicarbonato y el exceso de base se hace más negativo (de -3 a -5 en la Ciudad de México). La evaluación del estado ácido-base es de suma importancia en los pacientes críticamente enfermos.

El estándar de referencia para analizar el estado de oxigenación de un individuo es la GA. El parámetro más importante es la $\mathrm{PaO}_{2}$, cuando ésta es $<60 \mathrm{mmHg}$ se establece el diagnóstico de insuficiencia respiratoria (tipo I), la cual puede acompañarse de hipercapnia (tipo II). Cuando se documenta hipoxemia en la $\mathrm{GA}\left(\mathrm{PaO}_{2}<60\right.$ $\mathrm{mmHg}$ ) el clínico debe de analizar cuál es el potencial mecanismo de hipoxemia. En pocas ocasiones el mecanismo de hipoxemia es uno sólo, lo usual es que existan simultáneamente varios de ellos; analizarlos de manera individual en cada paciente ayuda en el conocimiento de la fisiopatología de la insuficiencia respiratoria. Los mecanis- mos de hipoxemia son: a) hipoxemia hipobárica (asociada a las alturas, también puede observarse en individuos que respiran aire hipóxico, como sucede en los incendios); b) hipoventilación (es el único mecanismo de hipoxemia que cursa con gradiente alveoloarterial de oxígeno normal, las principales causas son: hipoventilación central, defectos de caja torácica o enfermedades que afectan la función neuromuscular); c) desequilibrio $\mathrm{V}_{\mathrm{A}} / \mathrm{Q}$ (es el mecanismo más frecuente de hipoxemia); d) trastornos de la difusión de oxígeno (principalmente en las enfermedades que afectan el intersticio pulmonar); e) cortocircuito (alvéolos que reciben perfusión, pero se encuentran no ventilados), y f) desaturación de sangre venosa mixta (la sangre de la arteria pulmonar se encuentra desaturada lo que, cuando existe un estado hiperdinámico con tiempo de tránsito acortado como sucede en la etapa hiperdinámica de la sepsis, imposibilita que la sangre se oxigene apropiadamente durante su paso por el alvéolo).

El estado de oxigenación también puede ser evaluado mediante técnicas no invasivas. La oximetría de pulso es la forma más práctica, consiste en la medición del estado de oxigenación utilizando las propiedades diferenciales de absorción de la luz de la forma oxigenada y no oxigenada de la hemoglobina. Los oxímetros de pulso evalúan la transmisión de la luz roja e infrarroja a través de un tejido traslúcido con un adecuado flujo sanguíneo (idealmente el dedo o el pabellón auricular) y estiman el porcentaje de hemoglobina oxidada y reducida en el componente pulsátil de la señal. ${ }^{35}$ En otras palabras, durante cada pulsación existe un incremento transitorio del volumen de sangre arterial en el lugar de la medición, lo que produce mayor absorción de luz comparada contra la absorción basal también medida por el oxímetro. La absorción basal está dada por la piel, los tejidos blandos, el esmalte de uñas y la sangre venosa mientras que la absorción adicional, en la parte pulsátil, permite estimar de manera muy eficiente el componente arterial.

El oxímetro de pulso es utilizado para evaluar tendencias y no valores absolutos; sin embargo, las estimaciones que se realizan por medio de estos equipos suelen ser muy precisas y exactas. ${ }^{35}$ La variación documentada con saturaciones mayores de $90 \%$ es alrededor del $2.5 \%$, mientras que con saturaciones por debajo de $90 \%$ es cercana al $4 \%$. Debido a que no se conoce (más que por métodos de extrapolación) la exactitud y precisión de este instrumento con saturaciones por debajo de $70 \%$, las mediciones por debajo de estas cifras suelen no ser confiables.

Los factores que pueden afectar las mediciones del oxímetro de pulso son la pigmentación de la piel y tejidos (hiperbilirrubinemia) o la presencia de esmalte para las uñas; así como la hipotensión, ya que se pierde el componente pulsátil. Este componente también puede verse afectado por el movimiento. 
La interpretación de la oximetría es muy simple y los valores de referencia dependerán de la altitud sobre el nivel del mar. Para la Ciudad de México, en un estudio de base poblacional que incluyó a más de mil sujetos, la media de $\mathrm{SpO}_{2}$ fue de 92\%; no obstante, se considera normal cuando la $\mathrm{SpO}_{2}$ es mayor al $90 \%{ }^{34}$ Un aspecto a resaltar en la interpretación de la oximetría es que los oxímetros no pueden diferenciar la carboxihemoglobina y la oxihemoglobina debido a que en ambos casos, la hemoglobina se encuentra oxidada y esto hace que en condiciones de intoxicación por $\mathrm{CO}$ se obtengan mediciones supuestamente normales de $\mathrm{SpO}_{2}$ aun cuando exista hipoxemia grave. En consecuencia, una $\mathrm{SpO}_{2}$ normal no descarta hipoxemia, principalmente en fumadores o en personas con elevadas concentraciones de CO.

\section{PRUEBAS DE FUNCIÓN PULMONAR EN NIÑOS}

Las PFR en niños son de utilidad clínica rutinaria en enfermedades como el asma, fibrosis quística, displasia broncopulmonar y malformaciones congénitas, entre otras. Al igual que en los adultos, la mayor utilidad de la evaluación funcional radica en que pueden analizar la tendencia de la función pulmonar; es decir, se puede estudiar la función pulmonar de un individuo en relación con el tiempo y las intervenciones realizadas.

Las PFR en pediatría son muchas de ellas una adaptación de las pruebas utilizadas en los adultos, circunstancia que dista mucho de ser lo apropiado. ${ }^{36}$ Es necesario que las PFR en niños sean realizadas de manera estándar considerando la problemática que representa este grupo de edad, tanto en términos de cooperación para la realización de pruebas dependientes de esfuerzo como en las dificultades de interpretación ocasionadas por las modificaciones temporales (que no son lineales) de los parámetros funcionales secundarios al crecimiento y desarrollo. ${ }^{37}$ Los valores de referencia deben de ser generados a partir de valores medidos y en ninguna circunstancia deben utilizarse valores predichos mediante la técnica de extrapolación.

Como ejemplo de la falta de estandarización de las PFR en niños podemos mencionar el caso de la espirometría. Los criterios de calidad para considerar una espirometría como aceptable y repetible no son uniformes. La ATS en un documento exclusivo para PFR en niños propuso criterios que en general, podrían ser considerados como poco estrictos en comparación con lo señalado previamente por la misma sociedad en 2005, cuando recomendó que debían ser utilizados los mismos que en adultos a excepción del tiempo espiratorio. ${ }^{7}$ Datos preliminares de nuestro laboratorio muestran que el $75 \%$ de los niños de 4 a 6 años son capaces de realizar apropiadamente una espirometría bajo los criterios estrictos aplicados a población adulta; por lo tanto, nuestra recomendación está encaminada en el mismo sentido a lo propuesto por la ATS en 2005. ${ }^{7}$

La mayoría de las PFR pueden ser realizadas en escolares y adolescentes utilizando la estandarización empleada en adultos ${ }^{7} \sin$ embargo, en pacientes más pequeños las pruebas no se realizan de manera estándar, no existen valores de referencia, hay pobre disponibilidad de equipos y de personal especializado para la realización e interpretación de la prueba. Además, existe poco conocimiento por parte del clínico acerca de la utilidad de algunas pruebas.

La pletismografía y la dilución de gases son pruebas utilizadas para medir volúmenes y capacidades pulmonares. La pletismografía es la prueba más estandarizada. La babypletismografía requiere sedación, es aplicada en lactantes y no ha sido apropiadamente estandarizada. ${ }^{38}$ En el caso de las técnicas de dilución de gases, aunque no se requiere cooperación del paciente, son técnicas no estandarizadas y su uso está limitado a laboratorios especializados.

La medición de la resistencia de la vía aérea por técnica de interrupción (Rint) o de la impedancia (resistencia y reactancia) mediante la oscilometría de impulso son pruebas empleadas para evaluar la obstrucción bronquial. ${ }^{39,40}$ Tienen la ventaja de no ser dependientes del esfuerzo del enfermo y son realizadas a $\mathrm{VC}$ con equipos relativamente accesibles en términos de costo. Estas dos técnicas han sido estandarizadas y actualmente se trabaja en la generación de valores de referencia para niños mexicanos.

La prueba de $\mathrm{DL}_{\mathrm{co}}$ es poco utilizada en niños, es realizada bajo los estándares aplicados a adultos, lo que hace más imprecisa tanto la realización como la interpretación de la prueba. En aquellos en quienes se pueda realizar son aplicados los estándares utilizados en adultos.

La GA, la oximetría de pulso y la capnografía son pruebas ampliamente conocidas y existe mucha accesibilidad a las mismas.

La prueba de desaturación de oxígeno y la prueba de titulación de oxígeno suplementario son muy accesibles y estandarizadas en adultos; aunque realizadas frecuentemente en pediatría, no existe una recomendación específica en este grupo de edad. Una situación semejante sucede con otras pruebas como la medición de la presión inspiratoria y espiratoria máximas, prueba de reto bronquial con ejercicio, C6M y PCPE.

\section{OTRAS PRUEBAS DE FUNCIÓN RESPIRATORIA}

La medición de las presiones inspiratoria y espiratoria máximas $\left(\mathrm{Pi}_{\text {max }}\right.$ y $\mathrm{Pe}_{\text {max }}$, respectivamente) son pruebas bien toleradas y relativamente fáciles de realizar, permiten estimar la función neuromuscular del diafragma, así como de los músculos abdominales, intercostales y accesorios. ${ }^{41}$ En términos generales, la $\mathrm{Pi}_{\max }$ estima la fuerza de músculos 
inspiratorios (diafragma) y la $\mathrm{Pe}_{\max }$ la de los músculos abdominales e intercostales. Las pruebas consisten en que el paciente debe generar las máximas presiones inspiratorias y espiratorias contra una boquilla ocluida. La técnica estándar indica que la $\mathrm{Pi}_{\max }$ debe realizarse a partir de VR y la $\mathrm{Pe}_{\text {max }}$ de CPT. Hay que informar el valor máximo alcanzado en tres maniobras cuya variabilidad sea menor del 20\%. Estas pruebas son de utilidad clínica en enfermedades que afectan la función neuromuscular, tales como la distrofia muscular de Duchenne, distrofia muscular de Becker, atrofia espinal, lesión medular alta, síndrome de Guillain Barré, miastenia gravis, secuelas de poliomielitis o de enfermedad cerebrovascular, polimiositis y esclerosis lateral amiotrófica, entre otras. ${ }^{41} \mathrm{La} \mathrm{Pi}_{\max }$ y $\mathrm{Pe}_{\max }$ también pueden disminuir en cualquier otra enfermedad que afecte la posición normal del diafragma en la curva longitud tensión. Existen algunas contraindicaciones para la medición de la $\mathrm{Pi}_{\max }$ y $\mathrm{Pe}_{\max }$ que incluyen cirugía ocular u ótica reciente, angina inestable y cirugía abdominal reciente. La interpretación de la prueba debe hacerse con base en los valores predichos y en el límite inferior normal descrito para la población en estudio. En términos generales, es considerada anormal una $\mathrm{Pi}_{\max }$ o $\mathrm{Pe}_{\text {max }}$ menor del $60 \%$ del predicho. Al igual que para la mayoría de las PFR, estas pruebas son de gran utilidad cuando se realizan mediciones seriadas a lo largo del seguimiento clínico del paciente.

Las pruebas que evalúan el control de la respiración son rara vez necesarias en la práctica neumológica y tienen propósitos principalmente de investigación. Dichas pruebas permiten analizar la respuesta del sistema nervioso central a diversas condiciones a las que es sometido el sujeto. Algunas de ellas incluyen la prueba de apnea voluntaria, pruebas que evalúan la respuesta ventilatoria a hipoxemia e hipercapnia y la prueba de presión de oclusión $\mathrm{P}_{100}$ o $\mathrm{P}_{0.1}$ (presión generada a los 100 milesegundos de la inspiración). ${ }^{42}$

Para la medición de la resistencia de la vía aérea hay varias técnicas que incluyen a la rinomanometría, ${ }^{43}$ la Rint, ${ }^{40}$ la oscilometría de impulso ${ }^{39}$ o la medición de resistencia medida por pletismografía, etc. ${ }^{9}$ Los volúmenes pulmonares también se pueden medir por dilución de gases y por planimetría; pero el estándar de oro es la pletismografía. ${ }^{9}$ Existen pruebas sofisticadas para medir y cuantificar el desequilibrio $V_{A} / Q$, como la técnica de eliminación de múltiples gases inertes que tiene su principal aplicación en el contexto de la investigación. ${ }^{44}$ La polisomnografía es también una PFR dado que permite analizar detalladamente los patrones respiratorios durante el sueño así como el intercambio de gases. ${ }^{45}$

El análisis de gas exhalado no es intrínsecamente una PFR; pero dadas las implicaciones funcionales que pueden tener las incluimos de manera sucinta en este documento. En conjunto, estas pruebas consisten en medir biomarca- dores exhalados que suelen ser expresión de inflamación, los más estudiados son el óxido nítrico, el CO, el peróxido de hidrógeno, el $\mathrm{pH}$ y 8 -isoprostano, entre otros. ${ }^{46} \mathrm{La}$ medición de la fracción espirada de óxido nítrico (FeON) es la herramienta más estudiada y con mayor aplicación clínica. La Tabla 5 enuncia los factores que elevan o disminuyen la FeON. En la evaluación y seguimiento de pacientes con asma es en quienes la FeON es de mayor utilidad y en donde, además, se han obtenido resultados más consistentes. La determinación de la FeON es sencilla, no invasiva y de resultado inmediato. Los valores normales se sitúan entre 5 y 30 ppb. $^{46}$

Otros biomarcadores exhalados diferentes de FeON han sido estudiados y han surgido algunas propuestas para la estandarización de su medición mediante la obtención del condensado; no obstante, aún son técnicas en desarrollo debido a que las sustancias propuestas como biomarcadores son muy volátiles e inestables y requieren de un procesamiento prácticamente inmediato. Además, la tecnología utilizada para la medición de biomarcadores en condensado exhalado es cara y poco disponible. Cuando estas limitaciones hayan sido superadas y exista un mayor número de estudios clínicos, estas pruebas serán de gran utilidad para evaluar las condiciones de la vía aérea.

\section{PERSPECTIVAS Y CONCLUSIONES}

La evaluación funcional respiratoria es necesaria en el diagnóstico y seguimiento de la mayoría de los pacientes con enfermedades respiratorias crónicas; pero para que las PFR aporten información precisa y exacta deben realizarse de manera estandarizada, es decir, siguiendo procedimientos uniformes que minimicen las fuentes de variación. La práctica neumológica debe sustentarse en

Tabla 5: Factores o enfermedades que aumentan 0 disminuyen la fracción exhalada de óxido nítrico (FeON).

\begin{tabular}{|l|l|}
\hline Aumentan la FeON & Disminuyen la FeON \\
\hline Asma & Fibrosis quística \\
Exposición a aeroalergenos & Discinesia ciliar \\
Contaminación & Hipertensión arterial pulmonar \\
Apnea & Broncoconstricción \\
Broncodilatadores & Espirometría forzada (transitoria) \\
Bronquiectasias & Tabaquismo \\
Bronquitis crónica & Alcohol \\
Infecciones virales, & Cafeína \\
Tuberculosis & Menstruación \\
Rinitis alérgica & Nebulización con agua destilada \\
Bronquiolitis postrasplante & Neumonía \\
Alveolitis fibrosante & \\
Sarcoidosis pulmonar & \\
\hline
\end{tabular}


mediciones objetivas del funcionamiento pulmonar para lo cual, el clínico debe estar al tanto de qué prueba realizar y qué información se va a obtener a partir de dicha prueba. Algunas de las PFR pueden ser realizadas en el contexto del escrutinio; es decir, en la identificación de enfermedad en una población dada (usualmente en asintomáticos) o para evaluar la función pulmonar en pacientes que aquejan algún síntoma respiratorio. Estas pruebas incluyen la espirometría, la oximetría de pulso y la difusión pulmonar de CO. Otras PFR deben ser solicitadas con base en un análisis crítico de las posibilidades diagnósticas según la anamnesis y el examen físico, incluso, después de haber realizado algunas PFR consideradas como básicas, p. ej., la pletismografía corporal o la prueba cardiopulmonar de ejercicio deberían realizarse sólo cuando la espirometría o la $\mathrm{DL}_{\mathrm{CO}}$ no han sido suficientes en la evaluación funcional o cuando, una vez conocido el estado funcional, se desea investigar la presencia de alguna alteración específica. Tal es el caso de realizar una pletismografía para documentar hiperinflación una vez que la espirometría ha demostrado una alteración de tipo obstructivo, o bien para confirmar restricción pulmonar cuando la espirometría muestra disminución de la CVF.

$\mathrm{Si}$ consideramos que la espirometría, la $\mathrm{DL}_{\mathrm{CO}}$ la oximetría de pulso y la C6M son las pruebas que deberían ser catalogadas como básicas; luego entonces, deben hacerse esfuerzos para lograr que dichas pruebas estén ampliamente disponibles, especialmente para el especialista en medicina respiratoria. Para lograr dicha disponibilidad debemos analizar cada una de las pruebas. Los espirómetros son cada vez más precisos y exactos (mejores sensores de flujo) aun en el largo plazo y la estabilidad de los espirómetros ha sido confirmada en estudios epidemiológicos habiendo realizado miles de estudios. ${ }^{47}$ Los costos de los espirómetros también han disminuido (existen en México espirómetros hasta por el equivalente a 2,800 dólares estadounidenses), en donde hay que ser más cauteloso es en los valores de referencia espirométrica ya que eso puede generar errores graves de interpretación. En cuanto a la medición de la $\mathrm{DL}_{\mathrm{CO}^{\prime}}$ ésta es muy importante porque es una prueba estática de intercambio gaseoso. Aunque la medición de $\mathrm{DL}_{\mathrm{CO}}$ parecería ser compleja y poco disponible, existen esfuerzos tecnológicos que han logrado llevar al contexto de lo portátil, y a relativo bajo costo, los equipos de $\mathrm{DL}_{\mathrm{CO}}$. La C6M es muy sencilla y sólo requiere un pasillo de 30 metros, un oxímetro de pulso (idealmente), y seguir el procedimiento que ha sido aceptado de manera internacional. ${ }^{12}$

Creemos que el neumólogo debe ser firme promotor para que la espirometría, la $\mathrm{DL}_{\mathrm{CO}}$ y la C6M se popularicen. Estas pruebas deben estar disponibles en casi cualquier lugar y es responsabilidad del neumólogo hacer los esfuerzos necesarios para contar con dichas pruebas. Reconocemos que las limitaciones económicas podrían interferir con la disponibilidad de las pruebas; aun así, las ventajas de contar con dichas pruebas son abrumadoras. El especialista en medicina respiratoria debe también precisar en qué circunstancias o en qué momento tiene que referir a un paciente para realizar pruebas que no estén disponibles. La referencia a un laboratorio de fisiología pulmonar debe ser bien dirigida y solicitar pruebas que provean de la información apropiada para contestar a preguntas puntuales (por ejemplo: ¿cuál es la capacidad de ejercicio de este paciente?, ¿es este paciente portador de asma inducida por ejercicio?, ¿mi paciente con EPOC tiene hiperinflación estática o dinámica?, ¿cuál es el riesgo quirúrgico de mi paciente con EPOC?, etcétera).

Este manuscrito contribuye desde un punto de vista práctico con el conocimiento acerca de las PFR con el objetivo de facilitar al clínico, la decisión acerca de la prueba que mejor ayudará al diagnóstico y seguimiento de sus pacientes.

\section{REFERENCIAS}

1. Ruppel G. Manual of pulmonary function testing. 7th ed. St Louis, Missouri: Mosby; 1998.

2. Pellegrino R, Viegi G, Brusasco V, et ál. Interpretative strategies for lung function tests. Eur Respir J 2005;26:948-968.

3. Roca R, Rabinovich R. Clinical exercise testing. Eur Respir Mon 2005;31:146-165.

4. Hyatt RE, Scanlon PD, Nakamura M. Interpretation of pulmonary function tests. A practical guide. 3rd ed. Rochester, MN.: Lippincott Williams \& Wilkins; 2009.

5. Celli BR, MacNeeW; ATS/ERS Task Force. Standards for the diagnosis and treatment of patients with COPD: a summary of the ATS/ERS position paper. Eur Respir J 2004;23:932-946.

6. Crapo RO, Casaburi R, Coates AL, et ál. Guidelines for methacholine and exercise challenge testing-1999. This official statement of the American Thoracic Society was adopted by the ATS Board of Directors, July 1999. Am J Respir Crit Care Med 2000;161:309-329.

7. Miller MR, Hankinson J, Brusasco V, et ál. Standardization of spirometry. Eur Respir J 2005;26:319-338.

8. Vollmer WM, Gislason T, Burney P, et ál. Comparison of spirometry criteria for the diagnosis of COPD: results from the BOLD study. Eur Respir J 2009;34:588-597.

9. Wanger J, Clausen JL, Coates A, et ál. Standardization of the measurement of lung volumes. Eur Respir J 2005;26:511-522.

10. Casanova $\mathrm{C}$, Cote $\mathrm{C}$, de Torres JP, et ál. Inspiratory-to-total lung capacity ratio predicts mortality in patients with chronic obstructive pulmonary disease. Am J Respir Crit Care Med 2005;171:591-597.

11. Macintyre N, Crapo RO, Viegi G, et ál. Standardization of the singlebreath determination of carbon monoxide uptake in the lung. Eur Respir J 2005;26:720-735.

12. ATS statement: guidelines for the six-minute walk test. Am J Respir Crit Care Med 2002;166:111-117.

13. Enright PL, McBurnie MA, Bittner V, et ál. The 6-min walk test: a quick measure of functional status in elderly adults. Chest 2003;123:387-398.

14. Celli BR, Cote CG, Marin JM, et ál. The body-mass index, airflow obstruction, dyspnea, and exercise capacity index in chronic obstructive pulmonary disease. N Engl J Med 2004;350:1005-1012. 
Neumol Cir Torax. 2019; 78 (Supl 2): S81-S96

15. Callens E, Graba S, Gillet-Juvin K, et ál. Measurement of dynamic hyperinflation after a 6 -minute walk test in patients with COPD. Chest 2009;136:1466-1472.

16. Miyamoto S, Nagaya N, Satoh T, etál. Clinical correlates and prognostic significance of six-minute walk test in patients with primary pulmonary hypertension. Comparison with cardiopulmonary exercise testing. Am J Respir Crit Care Med 2000;161(2 Pt 1):487-492.

17. Singh S. Walking for the assessment of patients with chronic obstructive pulmonary disease. Eur Respir Mon 2007;40:148-164.

18. Martinez FJ, Han MK, Andrei AC, et ál. Longitudinal change in the BODE index predicts mortality in severe emphysema. Am J Respir Crit Care Med 2008;178:491-499.

19. Troosters T, Vilaro J, Rabinovich R, et ál. Physiological responses to the 6-min walk test in patients with chronic obstructive pulmonary disease. Eur Respir J 2002;20:564-569.

20. Cardoso F, Tufanin AT, Colucci M, Nascimento O, Jardim JR. Replacement of the 6-min walk test with maximal oxygen consumption in the BODE Index applied to patients with COPD: an equivalency study. Chest 2007;132:477-482.

21. Cahalin LP, Mathier MA, Semigran MJ, Dec GW, DiSalvo TG. The sixminute walk test predicts peak oxygen uptake and survival in patients with advanced heart failure. Chest 1996;110:325-332.

22. Swigris JJ, Wamboldt FS, Behr J, et ál. The 6 minute walk in idiopathic pulmonary fibrosis: longitudinal changes and minimum important difference. Thorax 2010;65:173-177.

23. Enright PL, Sherrill DL. Reference equations for the six-minute walk in healthy adults. Am J Respir Crit Care Med 1998;158:1384-1387.

24. Luna PE, Domínguez FME, Rodríguez PA, Gómez HJ. Estandarización de la prueba de caminata de 6 minutos en sujetos mexicanos. Rev Inst Nal Enf Resp Mex 2000;13:205-210.

25. Wise RA, Brown CD. Minimal clinically important differences in the six-minute walk test and the incremental shuttle walking test. COPD 2005:2:125-129.

26. Lacasse M, Maltais F, Poirier P, et ál. Post-exercise heart rate recovery and mortality in chronic obstructive pulmonary disease. Respir Med 2005;99:877-886.

27. Vargas-Domínguez $C$, Mejía-Alfaro R, Martínez-Andrade R, SilvaCerón M, Vázquez-García JC, Torre-Bouscoulet L. Prueba de desaturación y titulación de oxígeno suplementario. Recomendaciones y procedimiento. Neumol Cir Torax 2009;68:162-173.

28. King TE Jr. Clinical advances in the diagnosis and therapy of the interstitial lung diseases. Am J Respir Crit Care Med 2005;172:268-279.

29. Whipp BJ, Wagner PD, Agusti A. Determinants of the physiological systems responses to muscular exercise in healthy subjects. Eur Respir Mon 2007;40:1-35.

30. Ross RM. ATS/ACCP statement on cardiopulmonary exercise testing. Am J Respir Crit Care Med 2003;167:1451.

31. ERS Task Force, Palange P, Ward SA, et ál. Recommendations on the use of exercise testing in clinical practice. Eur Respir J 2007;29:185-209.
32. Wanger J, Crapo R, Irvin ChG. Pulmonary function laboratory management and procedure manual. Arterial blood gas analysis. American Thoracic Society; 2000.

33. Pérez Padilla JR, Vázquez García JC. Estimation of gasometric values at different altitudes above sea level in Mexico. Rev Invest Clin 2000;52:148-155.

34. Pérez-Padilla R, Torre-Bouscoulet $\mathrm{L}$, Muino A, et ál; Proyecto Latinoamericano de Investigación en Obstrucción Pulmonar (PLATINO) group. Prevalence of oxygen desaturation and use of oxygen at home in adults at sea level and at moderate altitude. Eur Respir J 2006;27:594-599.

35. Torre-Bouscoulet L, Chávez-Plascencia E, Vázquez-García JC, Pérez-Padilla R. Precision and accuracy of "a pocket" pulse oximeter in Mexico City. Rev Invest Clin 2006;58:28-33.

36. Beydon N, Davis SD, Lombardi E, et ál. An official American Thoracic Society/European Respiratory Society statement: pulmonary function testing in preschool children. Am J Respir Crit Care Med 2007;175:1304-1345.

37. Pérez-Padilla R, Regalado-Pineda J, Rojas M, et ál. Spirometric function in children of Mexico City compared to Mexican-American children. Pediatr Pulmonol 2003;35:177-183.

38. Davis SD. Neonatal and pediatric respiratory diagnostics. Respir Care 2003:48:367-384.

39. Meraz E, Nazeran H, Goldman M, Nava P, Diong B. Impulse oscillometric features of lung function: towards computer-aided classification of respiratory diseases in children. Conf Proc IEEE Eng Med Biol Soc 2008;2008:2443-2446.

40. Lombardi E, Sly PD, Concutelli G, etál. Reference values of interrupter respiratory resistance in healthy preschool white children. Thorax 2001;56:691-695.

41. Troosters T, Gosselink R, Decramer M. Respiratory muscle assessment. Eur Respir Mon 2005;31:57-71.

42. Calverley P. Control breathing. Eur Respir Mon 2005;31:44-56.

43. Schumacher MJ. Nasal dyspnea: the place of rhinomanometry in its objective assessment. Am J Rhinol 2004;18:41-46.

44. Wagner PD. The multiple inert gas elimination technique (MIGET). Intensive Care Med 2008;34:994-1001.

45. Kushida CA, Littner MR, Morgenthaler T, et ál. Practice parameters for the indications for polysomnography and related procedures: an update for 2005. Sleep 2005;28:499-521.

46. Silkoff PE, Erzurum SC, Lundberg JO, et ál. ATS workshop proceedings: exhaled nitric oxide and nitric oxide oxidative metabolism in exhaled breath condensate. Proc Am Thorac Soc 2006;3:131-145.

47. Pérez-Padilla R, Vázquez-García JC, Márquez MN, et ál. The longterm stability of portable spirometers used in a multinational study of the prevalence of chronic obstructive pulmonary disease. Respir Care 2006;51:1167-1171.

Los autores declaran no tener conflicto de intereses. 